\title{
Effects of Baihui electroacupuncture in a rat model of depression
}

\author{
Lin $\mathrm{Mao}^{1}$, Fei-Fei Lv ${ }^{2}$, Wen-Fu Yang ${ }^{3}$, Tian-Fang Zhang ${ }^{1}$, Zhong-Chun $\mathrm{Li}^{4}$, De-Qiang Li ${ }^{5}$, Zuo-Bing $\mathrm{Chen}^{1}$ \\ ${ }^{1}$ Department of Rehabilitation Medicine, The First Affiliated Hospital of Zhejiang University, Hangzhou, China; ${ }^{2}$ Department of Laboratory \\ Medicine, The First Affiliated Hospital of Zhejiang University, Hangzhou, China; ${ }^{3}$ Department of Breast Surgery, Shanxi Hospital of Oncology, \\ Taiyuan, China; ${ }^{4}$ Department of Neurology, Tongde Hospital of Zhejiang Province, Hangzhou, China; ${ }^{5}$ Department of Integrated Internal Medicine, \\ The First Affiliated Hospital, College of Medicine, Zhejiang University, Hangzhou, China \\ Contributions: (I) Conception and design: L Mao, DQ Li, ZB Chen; (II) Administrative support: None; (III) Provision of study materials or patients: \\ L Mao, FF Lv, WF Yang, TF Zhang, ZC Li, DQ Li; (IV) Collection and assembly of data: L Mao, FF Lv; (V) Data analysis and interpretation: L \\ Mao; (VI) Manuscript writing: All authors; (VII) Final approval of manuscript: All authors. \\ Correspondence to: Zuo-Bing Chen; De-Qiang Li. The First Affiliated Hospital, College of Medicine, Zhejiang University, No. 79, Qingchun Road, \\ Hangzhou, China. Email: czb1971@zju.edu.cn; 1dq5617@zju.edu.cn.
}

Background: This study aimed to investigate the effect of electroacupuncture (EA) on behavior in a rat model of chronic unpredictable mild stress (CUMS) and to explore the underlying molecular mechanisms.

Methods: A total of 45 adult male Sprague-Dawley rats were randomly divided into three groups: the control, CUMS, and CUMS plus EA groups. Rats in the CUMS and EA groups were subjected to a 3-week CUMS condition, while rats in the EA group received EA at the Baihui (GV 20) acupoint (2 Hz, $0.6 \mathrm{~mA})$ for $10 \mathrm{~min}$ once daily before being subjected to the CUMS condition. The sucrose preference test (SPT) was used as a measure to infer activation of the pleasure response to depression-like behaviour. After the behavioral test, 5-bromodeoxyuridine (BrdU) was intraperitoneally injected $(100 \mathrm{mg} / \mathrm{kg})$ and brain samples were collected $24 \mathrm{~h}$ later for the detection of hippocampal BrdU. Cell proliferation was determined according to the proportion of BrdU-positive cells. Brain-derived neurotrophic factor (BDNF) expression was detected.

Results: The severity of anhedonia, $\mathrm{BDNF}^{+}$cells, and $\mathrm{BrdU}^{+}$neurons in $\mathrm{DG}$ significantly decreased in CUMS rats, and was accompanied by a reduced BDNF and $\mathrm{BrdU}^{+}$expression $(\mathrm{P}<0.05)$. After EA, the low levels of $\mathrm{BDNF}^{+}$cells and $\mathrm{BrdU}^{+}$expression and the depression-like behavior increased markedly $(\mathrm{P}<0.05)$.

Conclusions: EA contributes to neuroprotection against CUMS by enhancing BDNF expression and improving hippocampal neurogenesis.

Keywords: Major depressive disorder (MDD); electroacupuncture (EA); chronic unpredictable mild stress (CUMS); brain-derived neurotrophic factor (BDNF); neurogenesis; rat

Submitted Sep 28, 2020. Accepted for publication Dec 16, 2020.

doi: 10.21037/atm-20-7459

View this article at: http://dx.doi.org/10.21037/atm-20-7459

\section{Introduction}

Major depressive disorder (MDD) is associated with poor social function, low quality of life, increased economic burden, and a high risk of suicide (1). Acupuncture has been used in China for thousands of years as a widely recognized alternative therapy in clinical practice, and has been used to treat various neurological diseases including depressive disorders (2-5). Due to recent advances in technology, a new form of acupuncture, electroacupuncture (EA), is now being applied in clinical practice. It has proved that EA could be used in improving cognitive and emotional symptoms in patients with organic brain diseases like Alzheimer's disease (6), anxiety disorders (7) and several physical diseases comorbid mood disorders including irritable bowel syndrome (8), hypertension (9) and stroke (10). Although exercise, electroconvulsive seizure therapy and transcranial magnetic stimulation are used to treat 
depression, psychopharmacological interventions are still the main stay of treatment options for most patients (11) by updating of evidence (12) and guideline $(13,14)$. Evidencebased medicine involving in the efficacy and tolerance on treatments of depression remains largely empirical. Several first-line pharmacological treatments are available, they are not effective for a significant proportion of patients (15) and concomitant adverse reactions limit the use in clinical practices (16). Acupuncture can activate a patient's own pain control system, stimulate blood flow, repair the physical damage of emotional distress, reduce pain, lift mood, and boost the immune system. If the combination of low-dose antidepressants plus EA could produce the same efficacy as solo antidepressants treatment with conventional dose, while reducing drug-related adverse reactions, this will be a relatively ideal treatment option. Evidences show that EA not only significantly improve depression symptoms and has fewer side effects than antidepressants (2) but when combined with a low dose of antidepressants mimic efficacy appear as compared to the same drug treatment with conventional dosage meanwhile showing lower incidences of adverse reactions and relapse (17). For these reasons, it has become a widely applied alternative therapy and is generating significant interest from Western countries (18). However, the antidepressant mechanism of EA is poorly understood, and evidence of its effectiveness remains weak (19). Further clinical and experimental studies are warranted to confirm the effectiveness of EA. Studies of animal models have indicated that EA exerts its neuroprotective effect against cerebral ischemia (20), hemorrhage (21), hypoxia, chronic stress (2), inflammation (22), and cognitive impairment (23) by upregulating the brain-derived neurotrophic factor (BDNF) expression in the hippocampus. Given that BDNF downregulation-induced hippocampal synaptic plasticity impairment plays an important role in the pathogenesis of MDD, the present study was undertaken to investigate the anti-depression-like effects of EA in rats exposed to chronic unpredictable mild stress (CUMS) and to explore the underlying mechanisms. Although different kinds of animal models of depression are used in laboratory experiments including surgical bilateral olfactory bulbectomy, maternal separation early life stress, learned helplessness, repeated restraint stress, social isolation, chronic social defeat stress, witness defeat, genetic and pharmacological intervene and CUMS (24), CUMS modal centers on the idea that low level chronic and unpredictable stressors, similar to what vulnerable individuals might experience in everyday life, could induce a wide range of behavioral deficits including decreased sucrose preference, motivation for reward stimuli and sexual behaviors along with increased aggression, anxiety-like behaviors and altered sleep patterns (25). The depression-like phenotype can be assessed with strong validity in deferent maze tests for cognition, open-field test for motivation and sucrose preference for anhedonia.

We present the following article in accordance with the ARRIVE reporting checklist (available at http://dx.doi. org/10.21037/atm-20-7459).

\section{Methods}

\section{Animals and grouping}

Forty-five adult male Sprague-Dawley rats weighing 200-240 g were purchased from the Animal Center of Zhejiang University and randomly divided into three groups: control $(\mathrm{n}=15)$, CUMS $(\mathrm{n}=15)$, and EA plus CUMS ( $\mathrm{n}=15$ ) using random number table method. After a baseline sucrose preference test (SPT), all rats except those in the control group were subjected to a 3 -week CUMS condition and housed individually. During this time, the EA group received daily EA at the Baihui acupoint. SPT was administered again at 7-, 14-, and 21-day intervals after the initiation of the CUMS condition to assess the severity of depression-like behavior. After the final SPT, 5-bromodeoxyuridine was intraperitoneally administered $(100 \mathrm{mg} / \mathrm{kg})$ to all animals. Twenty-four hours later the rats were killed with an overdose intravenous injection of $10 \%$ chloral hydrate at $3 \mathrm{~mL} / \mathrm{kg}$, and the bilateral hippocampi were collected for immunochemistry and western blotting. Experiments were performed under two projects the Traditional Chinese Drug Administration Bureau of Zhejiang Province (license No. 2017ZA083) and the Major Science and Technology Project in Medical and Health of Zhejiang Province (co-constructed Project by the Province and the Ministry, license No. WKJ-ZJ-1922) and granted by Animal Ethics Committee of Zhejiang University of Chinese Medicine (license No. 2016-327), in compliance with the guidelines of Zhejiang University for the care and use of animals.

\section{CUMS procedures}

Twenty-four hours after the baseline SPT, rats in the CUMS and EA plus CUMS groups were subjected to CUMS to induce behavior reflective of the core depression symptom: 
anhedonia. The CUMS protocol was designed to maximize the unpredictable stress according to previous reports $(26,27)$ with a minor modification. One of the following stressors was administered every day (in a random order) for 3 weeks: food deprivation for $20 \mathrm{~h}$; water deprivation for $17 \mathrm{~h}$; swimming at $4^{\circ} \mathrm{C}$ for $5 \mathrm{~min}$; experiencing at high temperature $\left(40^{\circ} \mathrm{C}\right)$ for $5 \mathrm{~min}$; remaining in the cage at a $45^{\circ}$ tilt for $17 \mathrm{~h}$; remaining on a shaking platform (horizontal shaking at high speed) for $10 \mathrm{~min}$; being restrained for $2 \mathrm{~h}$; remaining in a soiled cage (200 mL water mixed with $100 \mathrm{~g}$ sawdust bedding) for $5 \mathrm{~h}$; remaining in an environment with persistent bright light $17 \mathrm{~h}$; tail pinching for $2 \mathrm{~min}$; and exposure to unpleasant noise for $5 \mathrm{~min}$. Immediately after each stress session, rats were returned to their usual cage and maintained in standard conditions until the next stress session.

\section{EA treatment}

Acupuncture was performed as previously reported (22) at the Baihui (GV-20) acupoint in the EA group once daily during the 3-week CUMS exposure. GV-20 is located above the apex auriculate at which the fractional amplitude of low-frequency fluctuation could fully spread to mood relevant hippocampus and prefrontal cortex circuits (28). The sterilized disposable stainless steel needles $(0.3 \mathrm{~mm}$ in diameter) were inserted to $2-3 \mathrm{~mm}$, and EA was conducted for 10 min once daily with a dense-dispersed wave at a frequency of $2 \mathrm{~Hz}$ and a current of $1 \mathrm{~mA}$ (low frequency 5-channel TENS Unit and Electrical Needle Stimulator, Huatuo Medical Technology Co., Ltd., Suzhou, China).

\section{Sucrose preference testing}

The sucrose preference test (SPT) has been used as a measure of the severity of anhedonia, and details of the procedure have been described previously (29). In brief, animals were housed individually with two bottles containing either $1 \%$ sucrose solution or tap water with standard lab chow available continuously. At baseline, an initial SPT was performed before the CUMS condition, and then further SPTs were performed three times under similar conditions (two-bottle test, 2 -h periods) on every weekend after the commencement of the CUMS exposure.

\section{Hippocampal immunobistochemistry}

Immunohistochemistry was performed for the evaluation of BDNF or BrdU-positive cells. A SABC-kit (Wuhan
Boster Biological Technology, China) was applied to the immunohistochemistry for BDNF evaluation. After deparaffinization and hydration, sagittal sections $(5 \mu \mathrm{m})$ were blocked in 5\% albumin from bovine serum for $20 \mathrm{~min}$. After incubation with rabbit-anti-rat BDNF immunoglobin G (IgG) $(1: 200)\left(4{ }^{\circ} \mathrm{C}\right)$ overnight, sections were treated with biotinylated goat-anti-rabbit IgG and streptavidinbiotin peroxidase complex (SABC) for $2 \mathrm{~h}\left(37^{\circ} \mathrm{C}\right)$, followed by visualization with 3,3'-diaminobenzidine. Counterstaining was done with hematoxylin. This was followed by dehydration in alcohol, transparency in xylene, and mounting with neutral resin. The hippocampal neurogenesis was evaluated on the coronal sections $(5 \mu \mathrm{m})$. In brief, the sections were treated with $0.3 \%$ hydrogen peroxide in phosphate buffer saline (PBS) for $30 \mathrm{~min}$ and then with $10 \%$ normal goat serum in $0.05 \mathrm{M} \mathrm{PBS}$ for $30 \mathrm{~min}$. After incubation with anti-BrdU monoclonal antibody (1:200) overnight at room temperature, sections were exposed to SABC for $2 \mathrm{~h}\left(37^{\circ} \mathrm{C}\right)$. The sections were mounted in neutral resin after dehydration and then observed under an Olympus microscope. The $\mathrm{BDNF}^{+}$cells and $\mathrm{BrdU}^{+}$cells in the same region of the dentate gyrus were counted and analyzed with the FIVE software (Thermo Fisher Scientific).

\section{Western blotting}

Western blotting was performed as previously reported (30). Brain tissues were lysed in lysis buffer $(0.05 \%$ Igepal, $50 \mathrm{mM}$ Tris-HCl, $5 \mathrm{mM}$ EDTA, $150 \mathrm{mM} \mathrm{NaCl}, 1 \%$ deoxycholic acid, $0.1 \%$ SDS, $1 \%$ Triton X-100). The lysates were then centrifuged at $10,000 \mathrm{rpm}$ for $30 \mathrm{~min}$ at $4^{\circ} \mathrm{C}$, and the supernatants were harvested for the detection of protein concentration. Total proteins were separated on $12.5 \%$ SDS-polyacrylamide gels and then transferred onto a nitrocellulose membrane. Membranes were then blocked in PBS containing $0.1 \%$ Tween and $5 \%$ nonfat milk for $30 \mathrm{~min}$. Thereafter, membranes were treated with either anti-Cx43 monoclonal antibody (1:1,000, Sigma) or antiactin antibody (1:500, Sigma) overnight. After washing, membranes were incubated with SABC for $1 \mathrm{~h}$, followed by visualization with enhanced chemiluminescence. The expression of BDNF and BrdU was quantified by measuring the optical density (OD) at $260 \mathrm{~nm}$.

\section{Statistical analysis}

All statistical analyses were carried out with SPSS software 
version 15.0 (IBM SPSS Software), and all data are expressed as mean \pm standard deviation (SD). One-way analysis of variance (ANOVA) was used for between-group comparisons. A $\mathrm{P}$ value $<0.05$ was considered statistically significant.

\section{Results}

\section{Behavioral changes on SPT}

At baseline, there was no significant difference among

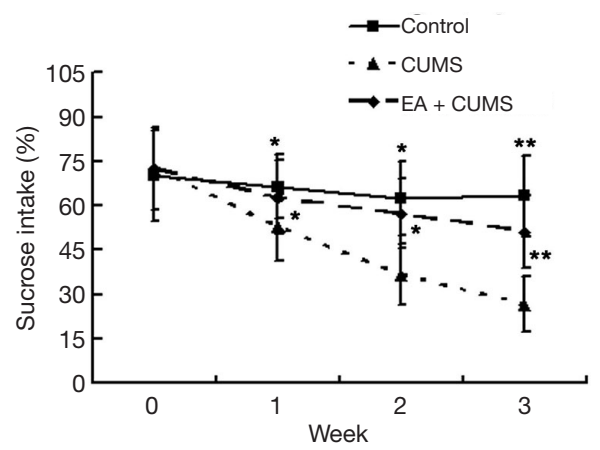

Figure 1 Sucrose intake in the three groups at different time points. One-way ANOVA, *, $\mathrm{P}<0.05$; ** $\mathrm{P}<0.01$ vs. CUMS group. ANOVA, analysis of variance; CUMS, chronic unpredictable mild stress; EA, electroacupuncture. the three groups in sucrose intake $\left[\mathrm{F}_{(2,42)}=0.47, \mathrm{P}>0.05\right]$. However, sucrose intake reduced significantly in the CUMS group compared to the control group after 1 week of stress exposure, and this effect lasted until the end of the experiment $(\mathrm{P}<0.05)$. After 1 week of CUMS exposure, sucrose intake was significantly different among the three groups $(\mathrm{P}<0.05)$, and EA treatment significantly increased the sucrose intake compared to the CUMS group at the same time point (all $\mathrm{P}<0.05)$ (Figure 1$)$.

\section{Change of BDNF-positive cells in DG}

BDNF-positive neurons had a large number of brown granules in the cytoplasm. These neurons were mainly found in the granular layer and nerve processes (Figure 2). The BDNF expression was at a high level in the control group but was significantly reduced in the CUMS group compared to the control group $(\mathrm{P}<0.05)$. After EA treatment, the BDNF expression in CUMS rats was significantly increased ( $\mathrm{P}<0.05$ vs. CUMS group).

\section{Neurogenesis in DG}

Newly generated neurons (BrdU positive) had dark brown granules in the nuclei. The presence of newly generated neurons suggests neurogenesis. The number
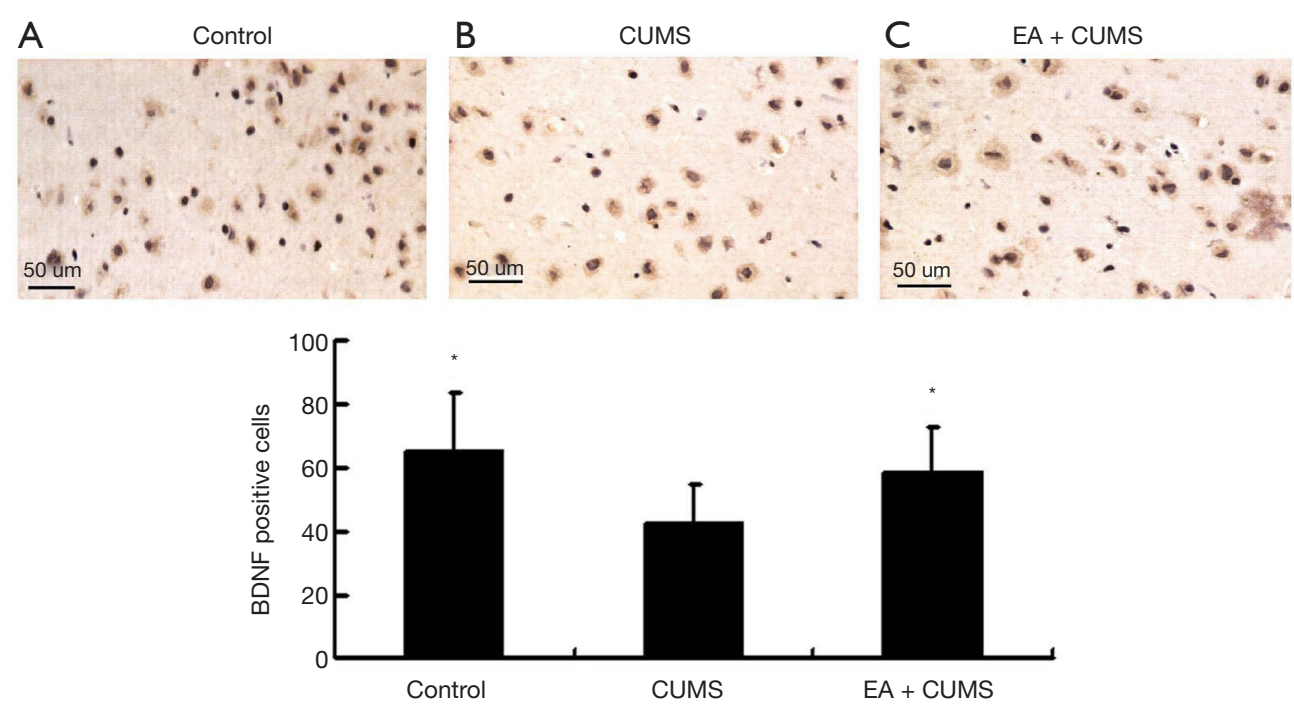

Figure 2 Immunohistochemistry for BDNF-positive cells (mean \pm SD) in DG of three groups. (A) Control group; (B) CUMS group; (C) EA plus CUMS group. BDNF ${ }^{+}$cells had brown granules in the cytoplasm around the black nuclei. *, $\mathrm{P}<0.05$ vs. CUMS group. $\mathrm{n}=15$, Scale bar $=50 \mu \mathrm{m}$. CUMS, chronic unpredictable mild stress; EA, electroacupuncture; BDNF, brain-derived neurotrophic factor; SD, standard deviation; DG, dentate gyrus. 

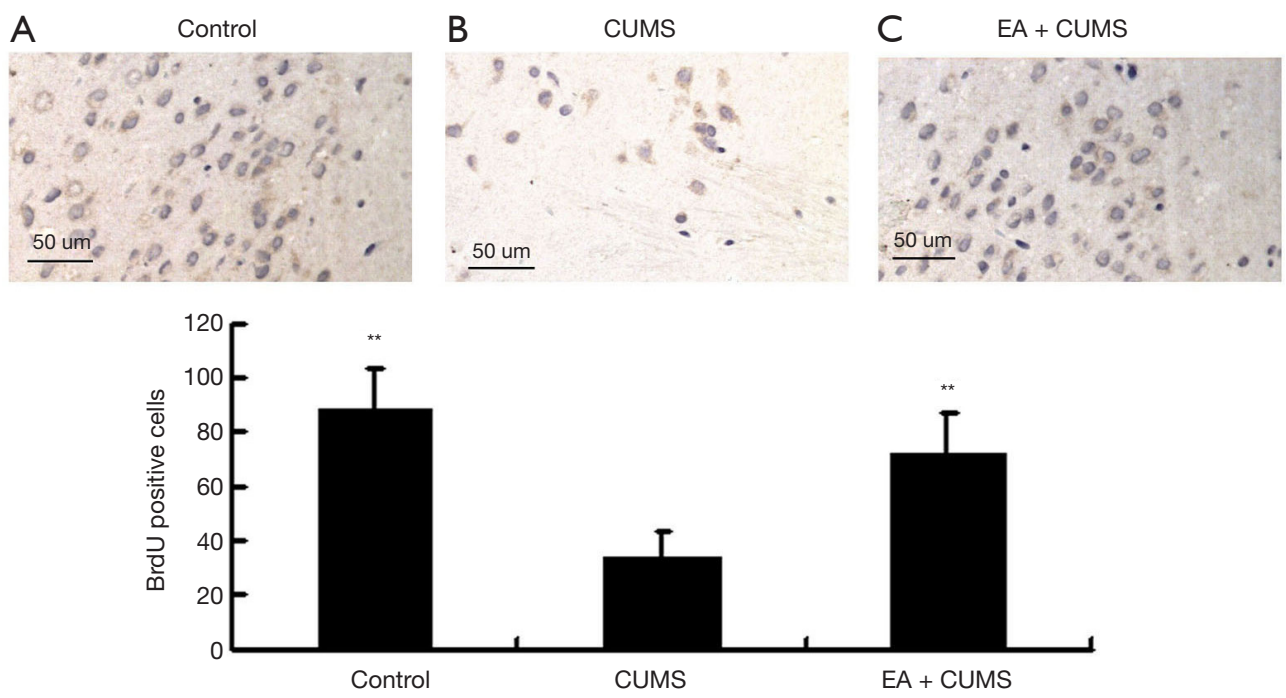

Figure 3 Immunohistochemistry for BrdU-positive cells (mean $\pm \mathrm{SD}$ ) in the DG $24 \mathrm{~h}$ after BrdU administration in the three groups. (A) Control group; (B) CUMS group; (C) EA plus CUMS group. **, $\mathrm{P}<0.01$ vs. CUMS group. $\mathrm{n}=15$, scale bar $=50 \mu \mathrm{m}$. CUMS, chronic unpredictable mild stress; EA, electroacupuncture; BrdU, 5-bromodeoxyuridine; SD, standard deviation; DG, dentate gyrus.

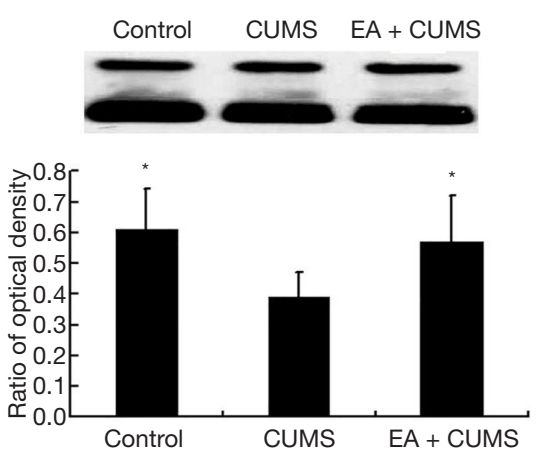

Figure $4 \mathrm{BDNF}$ protein expression (western blotting) in the different groups. Mean $\pm \mathrm{SD}$. * $\mathrm{P}<0.05$ vs. CUMS group. BDNF, brain-derived neurotrophic factor; CUMS, chronic unpredictable mild stress, EA, electroacupuncture.

of BrdU-positive cells in the DG of the hippocampus was significantly lower in the CUMS group than in the control group, but EA treatment significantly increased the BrdUpositive cells $(\mathrm{P}<0.05$ vs. CUMS group) (Figure 3).

\section{BDNF protein expression}

The protein expression of BDNF in the hippocampus of the CUMS rats was markedly lower than in the control group $(\mathrm{P}<0.05)$, but EA treatment significantly reversed the BDNF protein expression in CUMS-exposed rats (Figure 4).

\section{Discussion}

Investigations into the pathogenesis of MDD have made significant progress in recent times, with evidence suggesting that dysfunctional hippocampal neurogenesis and BDNF downregulation contribute to this disorder (31). It has reported exercise (32), antidepressants (33) and electroconvulsive seizure therapy (34) could reverse pathological synaptic remodeling and new born neuron formation modulated through BDNF elevation in depressed animals. In the CUMS model, animals may present with anhedonia, which is a core symptom of MDD (22), as indicated by their behavioral response to the SPT. In this study, the CUMS condition induced significant reduction in sucrose intake on the SPT, but EA treatment restored the sucrose intake in CUMS-exposed rats, suggesting that EA might also improve the symptoms of MDD. We further examined the mechanism underlying the neuroprotective effects of EA by evaluating hippocampal neurogenesis and BDNF expression. Results indicated that neurogenesis and the protein expression of BDNF was significantly reduced in rats exposed to the CUMS condition, which is consistent with previous findings that stress-induced depression is associated with decreased neurogenesis (35). A variety of studies have demonstrated the relationship between CUMS and reduced BDNF expression mediated by abnormal hippocampal plasticity (36). Research has confirmed 
combined Baihui and Dazhui acupuncture treatment may lead to a better therapeutic effect via co-regulation of neurotrophic factors in the prominent atrophic zones and promoting proliferation of neural progenitor cells in striatum in mice with ischemic stroke (37). Thus, the concomitant reduction in BDNF expression in rats exposed to CUMS in this study further confirms the role of $\mathrm{BDNF}$ in the pathogenesis of $\mathrm{MDD}$, and therefore treatments targeting improvement in BDNF expression in the brain may prove to be promising developments for neuroprotection in MDD (38).

The neuroprotective effect of EA at GV 20 in MDD rats may possibly be related to the improvement of neural circuitry in the case of mood disorders (39). Functional and structural brain imaging has revealed that the cortexhippocampal loop is associated with higher-order emotional and cognitive functioning (40), and stimulation at any acupoint including GV 20 in this loop may lead to the improvement of mood-related cerebral dysfunction. Furthermore, hippocampal neurogenesis is involved in the plasticity of hippocampal inner circuits. Neural progenitors in the DG originate from the subgranular layer. After proliferation, maturation, and migration to the CA3 area, the newly generated cells become mature and form a network with pyramidal neurons to integrate information into the cortex-hippocampal loop. In the growth and differentiation stage of neural stem cells, BrdU, an analogue of TTP that can be incorporated into DNA during the synthesis of DNA, has become a marker for newly generated cells, and the expression of BrdU therefore reflects the neurogenesis in local neural networks. A variety of factors are involved in the regulation of hippocampal neurogenesis including neurotransmitters, hormones, growth factors, and external environmental factors such as stress, social isolation, substance abuse, and physical exercise. A recent study reported that EA treatment after ischemic stroke may improve the post-stroke functional recovery by enhancing the proliferation and differentiation of neuronal stem cells via the BDNF signaling pathway (37).

CUMS reduces BDNF protein expression mainly via the dysfunctional hypothalamic-pituitary-adrenal (HPA) axis and the immune system (41). It is therefore possible that an increase in BDNF expression may exert a direct antioxidative effect (42) or indirectly improve the homeostasis of the neuroendocrine system (43) by inhibiting the hyperactivity of the HPA axis (44), decreasing the mRNA expression of adrenocorticotropic hormone (ACTH), a corticotropin-releasing hormone, in the hypothalamus and cortisol in the plasma, in order to control the immune response to tissue injury (45) and enhance the hippocampal serotonin system (46) which protects the brain against damage. This may partially explain why low-frequency EA is able to enhance mood and emotion, but further investigation is necessary to detail the exact mechanisms involved in EA and mood disorders.

Our study has several limitations. First, it must be cautious when the research conclusion is extended to human being duo to certain racial differences and limitations of animal model in rats. Second, the exact sites of current diffusion involving in emotional control regions in brain and the activation level of cell currents are not precisely studied. Finally, we only selected Baihui acupoint for treatment and this fact is not consistent with the real situations that based on the theory of meridians several acupuncture sites are always simultaneously used to treat patients to achieve wider stimulus fields and bigger efficacy. A further research will be performed to overcome these shortcomings in the future.

\section{Conclusions}

In conclusion, our study shows that administration of EA at the Baihui acupoint may improve the symptoms of MDD through increasing BDNF expression in the brain to restore hippocampal neurogenesis.

\section{Acknowledgments}

Funding: This study was supported by the Traditional Chinese Drug Administration Bureau of Zhejiang Province (No. 2017ZA083). This study was supported by grants from Major Science and Technology Project in Medical and Health of Zhejiang Province (co-constructed Project by the Province and the Ministry) (WKJ-ZJ-1922)

\section{Footnote}

Reporting Checklist: The authors have completed the ARRIVE reporting checklist. Available at http://dx.doi. org/10.21037/atm-20-7459

Data Sharing Statement: Available at http://dx.doi. org/10.21037/atm-20-7459

Conflicts of Interest: All authors have completed the ICMJE uniform disclosure form (available at http://dx.doi. 
org/10.21037/atm-20-7459). The authors have no conflicts of interest to declare.

Etbical Statement: The authors are accountable for all aspects of the work in ensuring that questions related to the accuracy or integrity of any part of the work are appropriately investigated and resolved. Experiments were performed under two projects the Traditional Chinese Drug Administration Bureau of Zhejiang Province (license No. 2017ZA083) and the Major Science and Technology Project in Medical and Health of Zhejiang Province (co-constructed Project by the Province and the Ministry, license No. WKJ-ZJ-1922) and granted by Animal Ethics Committee of Zhejiang University of Chinese Medicine (license No. 2016-327), in compliance with the guidelines of Zhejiang University for the care and use of animals.

Open Access Statement: This is an Open Access article distributed in accordance with the Creative Commons Attribution-NonCommercial-NoDerivs 4.0 International License (CC BY-NC-ND 4.0), which permits the noncommercial replication and distribution of the article with the strict proviso that no changes or edits are made and the original work is properly cited (including links to both the formal publication through the relevant DOI and the license). See: https://creativecommons.org/licenses/by-nc-nd/4.0/.

\section{References}

1. Mrazek DA, Hornberger JC, Altar CA, et al. A review of the clinical, economic, and societal burden of treatmentresistant depression: 1996-2013. Psychiatr Serv 2014;65:977-87.

2. Duan DM, Tu Y, Liu P, et al. Antidepressant effect of electroacupuncture regulates signal targeting in the brain and increases brain-derived neurotrophic factor levels. Neural Regen Res 2016;11:1595-602.

3. Fan L, Fu W, Chen Z, et al. Curative effect of acupuncture on quality of life in patient with depression: a clinical randomized single-blind placebo-controlled study. J Tradit Chin Med 2016;36:151-9.

4. Li ZX, Li Y, Yang WT, et al. The reporting quality of acupuncture for neurogenesis in experimental ischemic stroke study. Ann Transl Med 2019;7:123.

5. Yin X, Xu J, Dong B, et al. Efficacy and Safety of Electroacupuncture on Treating Depression Related Sleep Disorders: Study Protocol of a Randomized Controlled Trial. Evid Based Complement Alternat Med
2016;2016:1069597.

6. Feng Q, Bin LL, Zhai YB, et al. Long-term efficacy and safety of electroacupuncture on improving MMSE in patients with Alzheimer's disease Zhongguo Zhen Jiu 2019;39:3-8.

7. Amorim D, Amado J, Brito I, et al. Acupuncture and electroacupuncture for anxiety disorders: A systematic review of the clinical research. and Noneffectiveness of electroacupuncture for comorbid generalized anxiety disorder and irritable bowel syndrome. Complement Ther Clin Pract 2018;31:31-7.

8. Mak AD, Chung VCH, Yuen SY, et al. Noneffectiveness of electroacupuncture for comorbid generalized anxiety disorder and irritable bowel syndrome. J Gastroenterol Hepatol 2019;34:1736-42.

9. Chen H, Shen FE, Tan XD, et al. Efficacy and Safety of Acupuncture for Essential Hypertension: A Meta-Analysis. Med Sci Monit 2018;24:2946-69.

10. Zhan J, Pan R, Zhou M, et al. Electroacupuncture as an adjunctive therapy for motor dysfunction in acute stroke survivors: a systematic review and meta-analyses. BMJ Open 2018;8:e017153.

11. MacQueen G, Santaguida P, Keshavarz H, et al. Systematic Review of Clinical Practice Guidelines for Failed Antidepressant Treatment Response in Major Depressive Disorder, Dysthymia, and Subthreshold Depression in Adults. Can J Psychiatry 2017;62:11-23.

12. Dos Santos RG, Bouso JC, Alcázar-Córcoles MÁ, et al. Efficacy, tolerability, and safety of serotonergic psychedelics for the management of mood, anxiety, and substance-use disorders: a systematic review of systematic reviews. Expert Rev Clin Pharmacol 2018;11:889-902.

13. Gartlehner G, Gaynes BN, Amick HR, et al. Comparative Benefits and Harms of Antidepressant, Psychological, Complementary, and Exercise Treatments for Major Depression: An Evidence Report for a Clinical Practice Guideline From the American College of Physicians. Ann Intern Med 2016;164:331-41.

14. Kennedy SH, Lam RW, McIntyre RS, et al. Canadian Network for Mood and Anxiety Treatments (CANMAT) 2016 Clinical Guidelines for the Management of Adults with Major Depressive Disorder: Section 3. Pharmacological Treatments. Can J Psychiatry 2016;61:540-60.

15. Pandarakalam JP. Challenges of Treatment-resistant Depression. Psychiatr Danub 2018;30:273-84.

16. Tomlinson A, Efthimiou O, Boaden K, et al. Side effect profile and comparative tolerability of 21 antidepressants 
in the acute treatment of major depression in adults: protocol for a network meta-analysis. Evid Based Ment Health 2019;22:61-6.

17. Bai Y, Ouyang SL, Bai YJ, et al. Treatment for Persistent Somatoform Pain Disorder via Electroacupuncture and a Low Dosage of Fluoxetine Hydrochloride. Integr Med (Encinitas) 2017;16:28-31.

18. Wang X, Wang Z, Liu J, et al. Repeated acupuncture treatments modulate amygdala resting state functional connectivity of depressive patients. Neuroimage Clin 2016;12:746-52.

19. Sorbero ME, Reynolds K, Colaiaco B, et al. Acupuncture for Major Depressive Disorder: A Systematic Review. Rand Health Q 2016;5:7.

20. Zheng CX, Lu M, Guo YB, et al. Electroacupuncture Ameliorates Learning and Memory and Improves Synaptic Plasticity via Activation of the PKA/CREB Signaling Pathway in Cerebral Hypoperfusion. Evid Based Complement Alternat Med 2016;2016:7893710.

21. Li HQ, Li Y, Chen ZX, et al. Electroacupuncture Exerts Neuroprotection through Caveolin-1 Mediated Molecular Pathway in Intracerebral Hemorrhage of Rats. Neural Plast 2016;2016:7308261.

22. Tang WC, Hsu YC, Wang CC, et al. Early electroacupuncture treatment ameliorates neuroinflammation in rats with traumatic brain injury. BMC Complement Altern Med 2016;16:470.

23. Li W, Kong LH, Wang H, et al. High-frequency electroacupuncture evidently reinforces hippocampal synaptic transmission in Alzheimer's disease rats. Neural Regen Res 2016;11:801-6.

24. Ménard C, Hodes GE, Russo SJ. Pathogenesis of depression: Insights from human and rodent studies. Neuroscience 2016;321:138-62.

25. Hao Y, Ge H, Sun M, et al. Selecting an Appropriate Animal Model of Depression. Int J Mol Sci 2019;20:4827.

26. Mavrakis KJ, Wolfe AL, Oricchio E, et al. Genomewide RNA-mediated interference screen identifies miR19 targets in Notch-induced T-cell acute lymphoblastic leukaemia. Nat Cell Biol 2010;12:372-9.

27. Xu J, She Y, Su N, et al. Effects of Electroacupuncture on Chronic Unpredictable Mild Stress Rats Depression-Like Behavior and Expression of p-ERK/ERK and p-P38/P38. Evid Based Complement Alternat Med 2015;2015:650729.

28. Wang YY, Liu Z, Chen F, et al. Effects of acupuncture on craving after tobacco cessation: a resting-state fMRI study based on the fractional amplitude of low-frequency fluctuation. Quant Imaging Med Surg 2019;9:1118-25.
29. Stepanichev MY, Tishkina AO, Novikova MR, et al. Anhedonia but not passive floating is an indicator of depressive-like behavior in two chronic stress paradigms. Acta Neurobiol Exp (Wars) 2016;76:324-33.

30. Li DQ, Chen WX, Zhou YP, et al. Effect of ramipril on the regulation of the expression of connexins 40 and 43 in a rabbit model of arterial balloon injury. Mol Med Rep 2012;6:565-9.

31. Levy MJF, Boulle F, Steinbusch HW, et al. Neurotrophic factors and neuroplasticity pathways in the pathophysiology and treatment of depression. Psychopharmacology (Berl) 2018;235:2195-220.

32. Liu PZ, Nusslock R. Exercise-Mediated Neurogenesis in the Hippocampus via BDNF. Front Neurosci 2018;12:52.

33. Mondal AC, Fatima M. Direct and indirect evidences of BDNF and NGF as key modulators in depression: role of antidepressants treatment. Int J Neurosci 2019;129:283-96.

34. Kobayashi Y, Segi-Nishida E. Search for factors contributing to resistance to the electroconvulsive seizure treatment model using adrenocorticotrophic hormonetreated mice. Pharmacol Biochem Behav 2019;186:172767.

35. Pérez-Domper P, Palomo V, Gradari S, et al. The GSK-3inhibitor VP2.51 produces antidepressant effects associated with adult hippocampal neurogenesis. Neuropharmacology 2017;116:174-87.

36. Sawamoto A, Okuyama S, Amakura Y, et al. 3,5,6,7,8,3',4'-Heptamethoxyflavone Ameliorates Depressive-Like Behavior and Hippocampal Neurochemical Changes in Chronic Unpredictable Mild Stressed Mice by Regulating the Brain-Derived Neurotrophic Factor: Requirement for ERK Activation. Int J Mol Sci 2017;18:2133.

37. Kim YR, Ahn SM, Pak ME, et al. Potential benefits of mesenchymal stem cells and electroacupuncture on the trophic factors associated with neurogenesis in mice with ischemic stroke. Sci Rep 2018;8:2044.

38. Olesen MV, Wortwein G, Folke J, et al. Electroconvulsive stimulation results in long-term survival of newly generated hippocampal neurons in rats. Hippocampus 2017;27:52-60.

39. ACNP 55th Annual Meeting: Poster Session II December 6, 2016. Neuropsychopharmacology 2016;41:S289-454.

40. Gloveli T, Behr J, Dugladze T, et al. Kindling alters entorhinal cortex-hippocampal interaction by increased efficacy of presynaptic GABA(B) autoreceptors in layer III of the entorhinal cortex. Neurobiol Dis 2003;13:203-12.

41. Rieder R, Wisniewski PJ, Alderman BL, et al. Microbes and mental health: A review. Brain Behav Immun 
2017;66:9-17.

42. Kumar S, Mondal AC. Neuroprotective, Neurotrophic and Anti-oxidative Role of Bacopa monnieri on CUS Induced Model of Depression in Rat. Neurochem Res 2016;41:3083-94.

43. Zhu J, Chen Z, Zhu L, et al. Arginine Vasopressin and Arginine Vasopressin Receptor 1b Involved in Electroacupuncture-Attenuated Hypothalamic-PituitaryAdrenal Axis Hyperactivity in Hepatectomy Rats. Neuromodulation 2016;19:498-506.

44. Zhang H, Zhao G, Wang D, et al. Effect of electroacupuncture at different acupoints on hormones and neurotransmitters of hypotha-lamic-pituitary-adrenal

Cite this article as: Mao L, Lv FF, Yang WF, Zhang TF, Li ZC, Li DQ, Chen ZB. Effects of Baihui electroacupuncture in a rat model of depression. Ann Transl Med 2020;8(24):1646. doi: 10.21037/atm-20-7459 axis in rats under simulated weightlessness. Zhongguo Zhen Jiu 2015;35:1275-9.

45. Grech D, Li Z, Morcillo P, et al. Intraoperative Lowfrequency Electroacupuncture under General Anesthesia Improves Postoperative Recovery in a Randomized Trial. J Acupunct Meridian Stud 2016;9:234-41.

46. Le JJ, Yi T, Qi L, et al. Electroacupuncture regulate hypothalamic-pituitary-adrenal axis and enhance hippocampal serotonin system in a rat model of depression. Neurosci Lett 2016;615:66-71.

(English Language Editors: D. Fitzgerald and J. Gray) 